\title{
Electrophysiological indexes of illusory contours perception in humans
}

\author{
Alice Mado Proverbio ${ }^{\mathrm{a}, *}$, Alberto Zani ${ }^{\mathrm{b}}$ \\ a Department of Psychology, Laboratory of Cognitive Electrophysiology, University of Trieste, Via S. Anastasio 12, 34134 Trieste, Italy \\ ${ }^{\mathrm{b}}$ Electro-Functional Brain Imaging Unit, Institute of Neuroscience and Bioimaging, Consiglio Nazionale delle Ricerche, Via Fratelli Cervi 93, \\ 20090 Segrate, Milan, Italy
}

Received 18 December 2000; accepted 19 June 2001

\begin{abstract}
The present study investigated brain mechanisms underlying the perception of illusory contours, using recordings of event-related potentials of the brain (ERPs) in right-handed individuals. Forty different stimuli were presented randomly 1600 times in foveal vision; twenty of them produced the perception of illusory contours of a Kanizsa square, the remaining were obtained rotating outwards the inducers and they did not produce any illusory percept. Half of them had white inducers on a black background and vice versa; half of them were symmetrical and the other half asymmetrical. In lateral occipital areas illusory percepts produced larger evoked responses starting as early as $145 \mathrm{~ms}$ post-stimulus with the N1 peak. ERP data did not provide evidence of right-sided lateralisation of the processes underlying illusory contours formation at sensory level, as suggested by some neuroimaging and neuropsychological studies. The two cerebral hemispheres were differently activated while the subjective patterns formation progressed through neural processing stages. Indeed, brain response to illusory contours was more pronounced in the left occipital area at $\mathrm{N} 2$ component level (about $250 \mathrm{~ms}$ post-stimulus) and at right parietal sites at the latency of P300 component. Both background luminance and stimulus symmetry interacted with illusory boundaries formation. Present results confirm the hypothesis that the integration of contours arises at early stages of visual processing and highlight the primary role of edges continuity and boundary alignment in illusory contours perception. (C) 2001 Elsevier Science Ltd. All rights reserved.
\end{abstract}

Keywords: Event-related potentials (ERPs); Vision; Perception; Subjective figures; Kanizsa square; Hemispheric asymmetry

\section{Introduction}

Illusory contours are described as perceived edges that exist in the absence of local borders and that determine the perception of illusory or subjective figures, as the Kanizsa square (see [20,27] for a review). Subjective figures are often perceived as brighter (or darker, depending on background colour) than the surrounding surface $[15,16,33]$ and brightness seems to vary as a function of contour clarity or sharpness of illusory edges [21]. Other studies showed that the apparent increase in brightness of subjective figures and boundary contours completion are different phenom-

* Corresponding author. Tel.: + 39-040-676-2730; fax: + 39-040452-8022.

E-mail addresses: proverbi@univ.trieste.it (A.M. Proverbio), zani@carla.inb.mi.cnr.it (A. Zani). ena, depending on several factors [7,24,29]. Regions bounded by illusory contours are often perceived as nearer to the observer than the inducers, therefore mechanisms giving rise to depth perception are allegedly linked to illusory contours perception $[5,6,10]$.

A serious debate takes place in the literature about the possible involvement of primary visual area (area 17) in illusory contours perception and about the fact that the perception of illusory and real contours may activate overlapping visual regions. A recent fMRI study compared brain activation during vision of illusory and real contours, and highlighted the activation of lateral occipital areas V3A, V4v, V7 and V8 for both types of stimuli [23]. Furthermore, the Larsson et al. [19] PET study showed that the perception of real and illusory contours activated the same visual areas, with the exception of the right fusiform gyrus, which was specifically activated by perception of illusory contours. 
Ffytche and Zeki [8] showed in their study on regional cerebral blood flow (rCBF) that the perception of subjective contours in humans is associated with significant activity confined in early visual areas, particularly in area V2.

Single unit recording studies showed that cells of area 18 and not 17 respond to illusory contours in alert Macaca Mulatta monkeys [34]. More recently, cortical cells have been found in the extra-striate occipital areas signalling the presence of illusory defined boundaries in cats and monkeys [22]. According to the same study, cells with this property, although rare, were not totally absent in V1. Moreover, the use of stimuli which were different from those used in previous studies, allowed Grosof et al. [11] to find illusory contours responses in V1 neurons of macaque monkeys.

Hence there is evidence that illusory contour formation occurs within earlier centres of visual processing system [13,20], not necessarily involving higher order cortical areas, as it was originally suggested [9]. Yet many aspects of illusory defined boundary completion are still unclear because of inconsistency in the literature.

Another issue currently discussed concerns the existence of hemispheric lateralisation in the perception of illusory contours, distinguished from any brain asymmetry related to perceptual grouping and object segregation. Besides the right-sided activation of the fusiform gyrus recorded in the Larsson et al. [19] PET study, there seems to be further evidence of hemispheric asymmetry. For instance Hirsch et al. [14] in the fMRI study showed the predominance of brain activity related to illusory contours perception in the right extra-striate area. According to Hirsch et al. brain activation could involve striate cortex (area 17), too. Right hemispheric superiority was recorded in the behavioural task in which observers analysed the presence of subjective figures (Kanizsa squares). On the basis of the obtained results the authors concluded that the right hemisphere might have a special role in perceptual grouping and features conjunction [2]. Similarly, a neuropsychological study comparing right and left unilateral brain damaged patients demonstrated a right hemisphere involvement in the perception of subjective contour illusions [35].

A recent behavioural investigation carried out on two split-brain patients [4] did not provide evidence of right hemispheric lateralisation for illusory contours perception. The authors observed that while the two cerebral disconnected hemispheres were able to perceive illusory contours of a Kanizsa rectangle, the left hemisphere performed poorly in a condition in which pacmen were modified so that the perception of the illusory figure depended on amodal completion.
On the basis of these results they concluded that the right hemisphere is involved in perceptual grouping and not in illusory contours perception.

The above mentioned data highlight the inconsistency of hemispheric lateralisation when illusory boundaries formation is concerned. Recordings of brain electrical activity may be useful tools to investigate the presence of hemispheric asymmetries in cerebral responses during visual processing of illusory boundaries. Unfortunately, in the literature only a few electrophysiological studies provide data on brain mechanisms underlying illusory contour perception. Furthermore, in these studies the reduced number of electrodes used to collect VEP data does not guarantee reliable experimental proof of hemispheric asymmetry. Sugawara and Morotomi [31] recorded visual evoked potentials (from $\mathrm{CZ}, \mathrm{OL}$ and $\mathrm{OR}$ sites) to passively viewed illusory and control stimuli on a white or black background. Results showed an enhancement of N2 occipital deflection for subjective figures, with no effect of background on VEP amplitudes. The authors concluded that illusory contours formation takes place at higher levels in the visual system; the brightness contrast induced by the patterns is not sufficient to explain the N2 enhancement, since it occurred with figures both on a black and on a white background.

Korshunova [17] also recorded VEPs to Kanizsa squares and control configurations in a passive viewing task and discovered a difference in N180/P230 peak-to-peak amplitude and N300 latency at occipital $\mathrm{O} 1$ and $\mathrm{O} 2$ sites and the left posterior temporal site (T5) associated with subjective contours perception. Although interesting, currently available electrophysiological data did not allow any definitive conclusion about the discussed issue. In particular, the question of time course and of neurofunctional mechanisms underlying illusory contours perception remains still unsolved.

The present study is aimed at determining the time course of sensory and perceptual processing in the boundary completion process, analysing the timing of ERP responses. Secondly it aims at investigating the role of induced brightness in the perception of illusory contours comparing the evoked responses to patterns on a black background with responses to patterns on a white background. Thirdly the study aims at analysing the neuro-anatomical distribution of brain activity, computing ERP topographical maps. Last but not least, it is aimed at proving the existence of hemispheric lateralisation in brain response during illusory contours perception. Observers were asked to decide whether or not they had perceived an illusory square, pressing a button choosing between two alternatives. 


\subsection{Participants}

Eight college students (four females and four males) from the age of 18 to 24 took part to this study as paid volunteers. All students were right handed with right eye dominance and had normal or corrected-to-normal vision. None of them was familiar with the objective of the study.

\subsection{Stimuli}

Forty different black and white patterns subtending $2^{\circ}$ of visual angle were used as stimuli. Each pattern was presented randomly 40 times in the central visual field, namely 1600 stimuli were administered to each subject. Twenty of the 40 stimuli produced the illusory percept of a Kanisza square which subtended $1^{\circ} 45^{\prime}$ of visual angle ('Illusion' condition: I); the remaining were obtained through outwards rotation of the inducers and they did not produce any illusory percept ('no-Illusion' condition: nI). For each of the two categories, half of the patterns had white inducers on a black background ('Black') and the other half black inducers on a white ('White') background (see Fig. 1).

Each group of 40 stimuli included half 'Symmetrical' and half 'Asymmetrical' stimuli (see Fig. 1c). Stimuli were considered asymmetrical when the four inducers were irregular in shape (that is non geometrical) and different from one another. They were considered symmetrical when inducers were regular and almost identical in shape. Neither symmetrical or asymmetrical stimuli had boundaries nearby and parallel to the illusory contour, therefore they all produced a strong perceptual effect [1]. They all caused the subjective perception of an occluding figure (a square) superimposed on the inducing elements which appeared incomplete (see the review of Lesher [20] on inducer completeness).

Both stimuli with thick inducers (pacmen) and endline type stimuli were used (see Fig. 1a and b). Stimuli were flashed for $200 \mathrm{~ms}$ and the interstimulus interval (visual stimulus onset to onset) varied randomly between 800 and 1000 ms. Stimulus contrast was about $50 \%$. The fixation point was a small gray cross $(4 \mathrm{~mm}$ of size) at the centre of the TV monitor.

Stimuli of the two categories I and nI were designed to have the same amount of physical energy (luminance), since they consisted of the same elements presented in different orientations. The outwards rotation of external inducers determined the production of illusory boundaries, which also contributed to slightly change their Fourier spectra.

The Symmetry dimension was introduced in order to investigate the role of edges and their characteristics in boundary signals completion. The Background dimension was introduced to investigate the role of brightness enhancement in illusory contour perception.

The adoption of 40 different types of stimuli was to reduce negative effects of neurons adaptation and fatigue, consecutive images, after effects and the arousal decrement inherent to any repetitive flash stimulation paradigm.

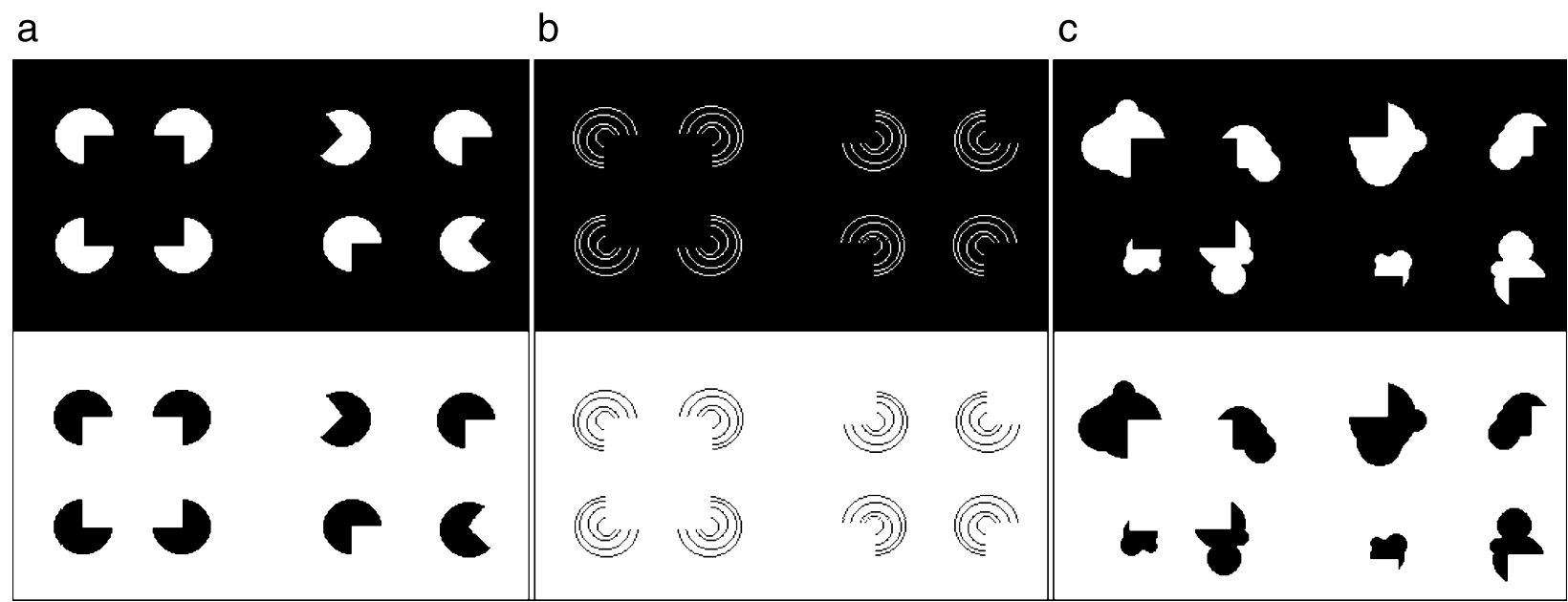

Fig. 1. Example of configurations adopted as stimuli in the present experiment. Stimuli displayed in the upper row belong to the categories Illusion (left) and no-Illusion (right) on a Black background (IB, nIB). In the lower row there are stimuli belonging to the categories Illusion (left) and no-Illusion (right) on a White background (IW, nIW). (a) Stimuli generated by thick inducers (pacmen) developing the classical Kanizsa square illusion in the condition in which inducers are rotated inwards. These types of stimuli belong to the category of Symmetric patterns, because the outer part of the inducers is symmetrical and regular. (b) Example of stimuli generated by line-ends. Inducers consist of pseudo-concentric rings with missing arcs. These kinds of stimuli are symmetrical, too. (c) Example of stimuli generated by irregular inducers, to investigate the role of stimulus symmetry in detecting illusory contours. Stimuli belonging to this class are defined as asymmetric patterns, because the outer part of the inducers is asymmetrical and irregular. 


\subsection{Procedure}

Participants were seated comfortably in a darkened, acoustically and electrically shielded box. A high resolution VGA computer screen was positioned at a distance of $114 \mathrm{~cm}$ from their eyes outside the box. They were asked to fix the centre of the screen and to avoid any eye or body movement during the recording session. The task consisted in responding as accurately and as fast as possible to all stimuli judging whether they had subjectively perceived an illusory square. Participants responded pressing a button with the index finger to I stimuli and with the middle finger to $\mathrm{nI}$ stimuli. Left and right hands were used alternatively during all sessions. The aim of the behavioural task was to make the observer draw their attention to perceived images, since stimuli were flashed at a relatively fast rate.

Each session consisted of 16 blocks of trials. In half of them only patterns on a white background were presented and vice-versa in the other half. Each block consisted of 103 trials of stimuli and lasted about 3 minutes. The first three visual stimuli were warning signals (SET, READY, GO!) inviting viewers to concentrate and to stare the fixation cross. After each run, a pause of several minutes followed. Several practice trials were carried out to let the participants get used to the different configurations. The order of stimuli presentation, response hand and background colour were counterbalanced among subjects.

\subsection{EEG recording}

The electroencephalogram (EEG) was continuously recorded from 28 scalp sites using tin electrodes mounted in an elastic cap (Electro-cap). Electrodes were positioned at frontal (Fp1, Fp2, FZ, F3, F4), central (CZ, C3, C4), temporal (T3, T4), posterior-temporal (T5, T6), parietal (PZ, P3, P4), and occipital scalp sites $(\mathrm{O} 1, \mathrm{O} 2)$ of the International 10-20 System. Additional electrodes were placed at half the distance between homologous posterior-temporal and occipital sites (OL, OR), half the distance between $\mathrm{O} 1$ and $\mathrm{O} 2$ sites (OZ), 10\% of nasion-inion distance below $\mathrm{OZ}$ (INZ), 10\% of nasion-inion distance above OZ (POZ), $10 \%$ to the left and the right of POZ site (PO1, PO2), 10 and $20 \%$ respectively to the left and right of INZ site (IN1, IN2, IN3, IN4).

Horizontal and vertical oculograms (EOG) were recorded to guarantee fixation maintenance. Blinks and vertical eye movements were monitored through two electrodes placed below and above the right eye; horizontal movements were recorded through electrodes placed at the outer canthi of the eyes. Linked earlobes were used as reference lead. The EOG and the EEG were amplified with a half-amplitude band pass of
$0.1-70 \mathrm{~Hz}$ and $0.01-70 \mathrm{HZ}$, respectively. Electrode impedance was kept below $5 \mathrm{~K} \Omega$. Continuous EEG and EOG were digitised at a rate of 512 samples/s.

All trials contaminated with eye or body movements were rejected. ERPs were averaged offline from $100 \mathrm{~ms}$ before probe presentation to $1000 \mathrm{~ms}$ after probe presentation. ERP trials associated with an incorrect behavioural response were also excluded from further analysis. For each subject, distinct ERP averages were obtained as a function of stimulus category. The major ERP components were identified and measured automatically by a computer program with reference to the baseline voltage averages over the interval from -100 to $0 \mathrm{~ms}$. ERP deflections were labelled according to a polarity-latency convention and quantified measuring peak latency and baseline-to-peak amplitude values within a specific latency range, centred approximately on the peak latency of the deflection seen in the grand average waveforms.

The occipital P1, peaking at about $86 \mathrm{~ms}$, was defined as the most positive deflection, between 70 and $120 \mathrm{~ms}$ post-stimulus at homologous P3, P4, T5, T6, O1, O2, OL, OR, PO1, PO2, IN3, IN4 recording sites. The occipital N1, peaking at about $146 \mathrm{~ms}$, was defined as the most negative deflection, between 125 and 175 $\mathrm{ms}$ at the same electrode sites. The posterior N2, peaking at about $250 \mathrm{~ms}$, was defined as the most negative deflection, between 200 and $300 \mathrm{~ms}$ at the same electrode sites.

Anterior and posterior P300 peaks were identified because of the different response patterns defined as functions of background luminance. P300 measures recorded at anterior and posterior sites underwent two different ANOVAs because of the high number of electrode sites.

Posterior P300, peaking at about $390 \mathrm{~ms}$ was defined as the most positive deflection, between 300 and $500 \mathrm{~ms}$ at homologous P3, P4, T5, T6, PO1, PO2 recording sites. Anterior P300 peaking at about $405 \mathrm{~ms}$ was defined as the most positive deflection, between 300 and $500 \mathrm{~ms}$ at anterior F3, F4, C3, C4, T3, T4 recording sites.

ERP components were also measured at midline sites and data were submitted to separate analyses of variance. $\mathrm{P} 1, \mathrm{~N} 1$ and $\mathrm{N} 2$ deflections were measured at $\mathrm{Pz}$, $\mathrm{POz}, \mathrm{Oz}$ and $\mathrm{INz}$ sites. $\mathrm{P} 3$ was measured at $\mathrm{Fz}, \mathrm{Cz}, \mathrm{Pz}$, $\mathrm{Oz}$ and INz electrode sites.

\subsection{Data analysis}

A computerised artifact rejection and baseline correction procedure was performed before averaging. Grand-average ERPs were separately computed for each stimulus type as a function of the following factors: 'illusion' (illusion $=\mathrm{I}, \quad$ no-illusion $=\mathrm{nI}$ ), 'background' $\quad($ white $=\mathrm{W} ; \quad$ black $=\mathrm{B}), \quad$ 'symmetry' 
( symmetric $=\mathrm{S}$; asymmetric $=\mathrm{aS})$. For each of the eight subjects and 28 scalp recording channels, eight different ERP waveforms were obtained by averaging about 160 trials. They were labelled IWS, IWaS, IBS, IbaS, nIWS, nIWaS, nIBS and nIbaS.

ERP amplitude and latency measures were analysed with repeated-measure five ways ANOVAs, separately for each ERP component of interest. Factors were 'illusion', 'background', 'symmetry', 'electrode site' (depending on the ERP component considered) and 'cerebral hemisphere' (left and right). Additional ANOVAs were carried out on ERP measures recorded at midline sites. The same factors were used, with the exception of factor 'hemisphere', which was omitted.

Topographical voltage maps of ERP components were obtained by plotting colour-coded iso-potentials derived from interpolation of voltage values between scalp electrodes at specific latencies.

Percentages were transformed in arcsine values and submitted to a four way ANOVA for each subject error and miss. Reaction times to target stimuli not exceeding 2 S.D. above or below the mean were also submitted to a four way ANOVA. For each of the above mentioned behavioural measures, the following ANOVA factors were adopted 'illusion', 'background', 'symmetry', and 'response hand' (left and right).

Greenhouse-Geisser corrections were employed for both behavioural and electrophysiological analyses to reduce positive bias resulting from repeated factors with more than two levels. Post-hoc Tukey tests were carried out for multiple means comparisons.

\section{Results}

\subsection{Behavioural data}

Correct answers reached $95.16 \%$, which means that stimuli produced a reliable illusion effect.

Reaction times to illusory patterns $(450 \mathrm{~ms})$ were faster than to non illusory patterns $(489 \mathrm{~ms})$, as the significance of Illusion factor $(F(1,7)=11.55 ; P<0.01$; $\varepsilon=1)$ demonstrates. It may be assumed that the viewer was more certain when deciding on the presence rather than the absence of the illusory square. When inducers were asymmetrical (see Fig. 1c as an example of these stimuli), it was easier to exclude than to ascertain the presence of the illusory square, i.e. to respond No rather than Yes, which is confirmed by the results of ANOVA performed on arcsine-transformed error percentages, which yielded a significant triple interaction of Background $\times$ Symmetry $\times$ Illusion $(F(1,7)=5.9 ; \quad P<$ $0.05 ; \varepsilon=1)$. Post-hoc comparisons for illusory asymmetrical patterns showed higher percentages of wrong categorisations than for non illusory asymmetrical patterns. Table 1 shows that the overall error rate for
Table 1

Mean percentage of errors made by observers in task performance, as a function of stimulus type and background luminance

\begin{tabular}{llllll}
\hline Symmetry illusion & \multicolumn{2}{l}{ Symmetrical } & & \multicolumn{2}{l}{ Asymmetrical } \\
\cline { 6 - 6 } \cline { 5 - 6 } & $\mathrm{I}$ & $\mathrm{nI}$ & & $\mathrm{I}$ & $\mathrm{nI}$ \\
\hline White & 4.06 & 4.81 & & 5.37 & 3.81 \\
S.D. & 0.7 & 0.8 & & 0.9 & 0.6 \\
Black & 3.12 & 3.68 & & 4.37 & 3.25 \\
S.D. & 0.8 & 0.7 & & 0.8 & 0.7 \\
\hline
\end{tabular}

stimuli on a white background was higher than for stimuli on a black background, although post-hoc comparisons were significant only at $t$ test level $(P<0.05)$.

\subsection{Electrophysiological data}

\subsubsection{P1 deflection}

3.2.1.1. Posterior $P 1$. This positive response was larger at lateral occipital sites as shown in post-hoc comparisons $(P<0.01)$ performed for the main factor Electrode $(F(5,35)=3.85 ; \quad P<0.007 ; \varepsilon=0.41$, correct. $F(2,14)$; $P<0.05)$. The significant interaction of Electrode $\times$ Hemisphere $(F(5,35)=2.89 ; P<0.03 ; \varepsilon=0.41$, correct. $F(2,14) ; P<0.05)$ showed that $\mathrm{P} 1$ was larger at right lateral occipital sites, as it often results in visual perception paradigms. The triple interaction of Symmetry $\times$ Electrode $\times$ Hemisphere was significant $(F(5,35)=3.43$; $P<0.01 ; \varepsilon=0.41$, correct. $F(2,14) ; P<0.05)$, too. Posthoc comparisons showed that $\mathrm{P} 1$ to symmetrical configurations was larger than $\mathrm{P} 1$ to asymmetrical configurations at the right lateral-occipital site $(P<$ $0.01)$, where this component reached its maximum amplitude (see Fig. 2).

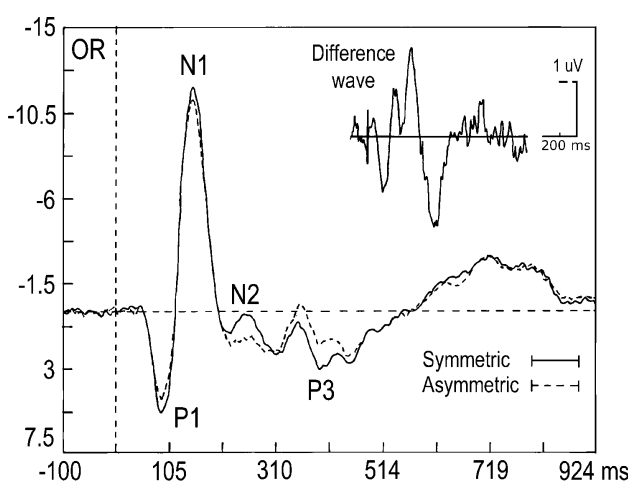

Fig. 2. Grand-average ERPs recorded at the right lateral occipital site in response to symmetrical or asymmetrical patterns independent of other factors. Stimulus symmetry produced larger P1, N1, N2 and P3 evoked responses: these effects are clearly observable in the right upper corner, where the difference wave obtained subtracting ERPs to asymmetrical from ERPs to symmetrical patterns is shown. 


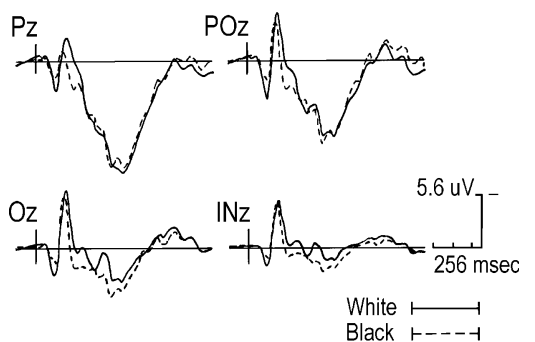

Fig. 3. Effect of background. Grand-average ERPs recorded at posterior midline sites in response to patterns with black or white background. The amplitude of evoked sensory responses was greater when patterns were presented on a white background.

\subsubsection{Midline P1. The ANOVA performed on $\mathrm{P} 1$} measures recorded at midline sites $(\mathrm{PZ}, \mathrm{POz}, \mathrm{Oz}, \mathrm{Inz})$ resulted in the interaction of Background $\times$ Electrode $(F(3,21)=3.29 ; \quad P<0.05 ; \quad \varepsilon=0.84$, correct. $\quad F(3,18)$; $P<0.05)$ with $\mathrm{P} 1$ to figures with white background $(P<0.01)$ at $\mathrm{POZ}$ and $\mathrm{Pz}$ electrode sites (see Fig. 3) being larger. This result might depend on the fact that patterns on a white background were brighter than those on a black background, basically reflecting the strength of photoceptors stimulation. We believe that this was due to the fact that stimuli were presented on a computer screen in a dimly lit room and not on paper in daylight. Moreover observers did not subjectively perceive figures on a black background as darker than the surrounding surface. Indeed, it has been shown that the apparent change in brightness (brightness or darkness enhancements) of subjective figures decreases according to decreasing levels of illumination and may be absent at low luminance level [30].

Hence in this latency range (between 70 and $120 \mathrm{~ms}$ post-stimulus) brain responses were affected by stimulus symmetry and background luminance and not by illusory contours.

\subsubsection{N1 deflection}

\subsubsection{Posterior N1. The ANOVA performed on N1} amplitude showed as in the case of occipital P1 that this component (peaking at about $146 \mathrm{~ms}$ ) was larger at lateral occipital sites, as confirmed by post-hoc tests $(P<0.01)$ performed for the significant factor Electrode $(F(5,35)=9.28 ; \quad P<0.0000 ; \quad \varepsilon=0.33$, correct. $F(2,12) ; P<0.005)$. The significant interaction of Electrode $\times$ Hemisphere $(F(5,35)=3.34 ; P<0.01 ; \varepsilon=0.51$, correct. $F(3,18) ; P<0.05)$ also indicated a right hemispheric lateralisation of $\mathrm{N} 1$ response which was larger at right lateral occipital site $(P<0.01)$. The interaction between Illusion and Electrode was significant, too $(F(5,35)=2.62 ; \quad P<0.05 ; \quad \varepsilon=0.75$, correct. $F(4,25)$; $P<0.05)$. Post-hoc comparisons showed that N1 to patterns eliciting an illusory percept (I vs. nI) was larger at lateral, posterior occipital and posterior temporal electrode sites $(P<0.01)$. This result suggests that the lateral occipital area responded bilaterally to illusory defined subjective figures as early as $145 \mathrm{~ms}$ post-stimulus (see Fig. 4). The lack of any significant interaction between Hemisphere and Illusion factors does not confirm the existence of a lateralisation of sensory processes underling illusory contour perception.

Analysis of ERP topographical distribution (see maps of Fig. 5a), as a function of time course, showed that boundary completion processes activated the lateral occipital areas (probably corresponding to extrastriate cortex) as early as $80 \mathrm{~ms}$ at P1 level, although this effect was not statistically significant. The later increase in amplitude of N1 response was also observable over the same brain regions, with bilateral activation.

3.2.2.2. Midline N1. The ANOVA performed for midline posterior electrodes, confirmed the early effect of boundary completion at N1 level. The Illusion $\times$ Electrode interaction $(F(3,21)=3.2 ; P<0.05 ; \varepsilon=0.84$, correct. $F(3,18) ; P<0.05)$ showed that $\mathrm{N} 1$ to I stimuli was larger than to $\mathrm{nI}$ stimuli at $\mathrm{Oz}$ and $\mathrm{INz}$ electrode sites $(P<0.01)$. Table 2 shows mean amplitudes of $\mathrm{N} 1$ deflection for this statistical comparison. The ANOVA also yielded a main effect of Electrode $(F(3,21)=9.54$; $P<0.0005 ; \varepsilon=0.52$, correct. $F(2,11) ; P<0.005)$ with $\mathrm{N} 1$ being larger at $\mathrm{POz}$ and $\mathrm{Oz}$ sites $(P<0.01)$. Another interesting result is the effect of stimulus Symmetry or regularity (independent of illusion formation) interacting with the Electrode factor $(F(3,21)=8.2$; $P<0.001 ; \varepsilon=0.62$, correct. $F(2,13) ; P<0.01)$. Indeed, $\mathrm{N} 1$ response to symmetrical configurations was larger than to asymmetrical configurations at the inion electrode sites $(P<0.05)$. Fig. 6 shows grand-average ERPs recorded at midline sites $\mathrm{POz}$ and Inz, and displays the effect of stimulus symmetry on N1 amplitude in the inion area, absent in the parietal-occipital area.

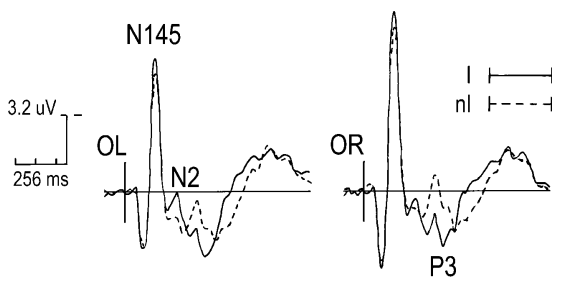

Fig. 4. Effect of illusory contours. Grand-average ERPs recorded at left and right lateral occipital sites (OL and $\mathrm{OR}$ ) in response to stimuli which induced (I) or did not induce (nI) the perception of illusory contours. The data indicate that illusory contours formation was associated with bilateral activation of lateral occipital cortex followed by significant left-sided activation. 


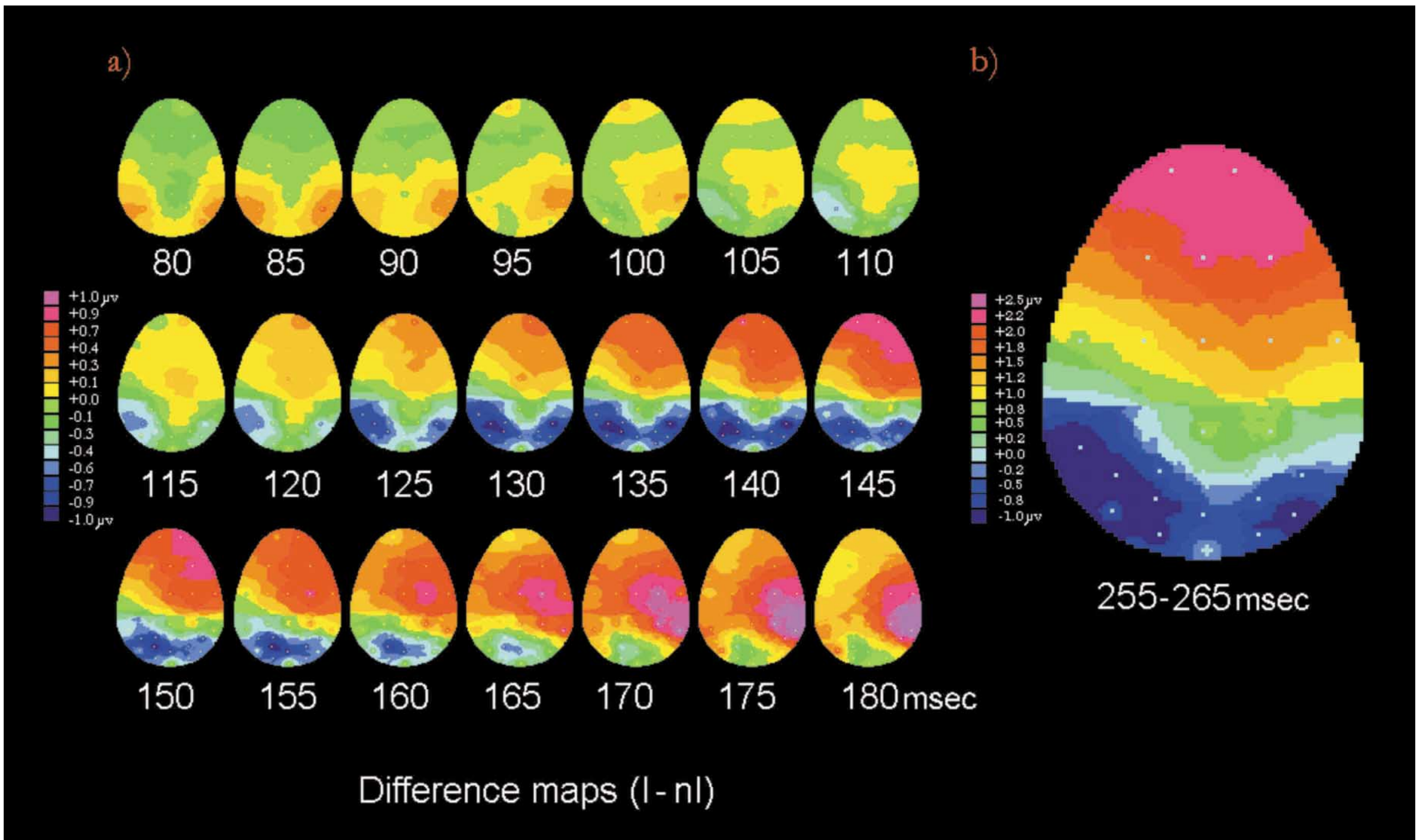

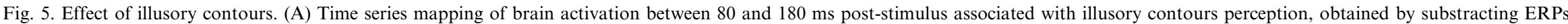

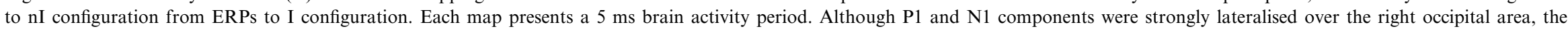

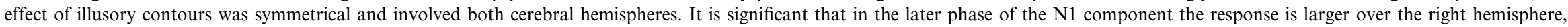

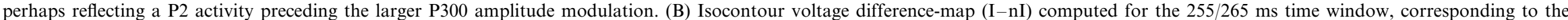
peak of the effect at N2 level. 
Table 2

N1 mean amplitude $(\mu \mathrm{V})$ recorded at posterior midline sites as a function of illusion condition

\begin{tabular}{|c|c|c|c|c|c|c|c|c|}
\hline \multirow[t]{2}{*}{ Electrode illusion } & \multicolumn{2}{|l|}{$\mathrm{Pz}$} & \multicolumn{2}{|l|}{$\mathrm{Poz}$} & \multicolumn{2}{|l|}{$\mathrm{Oz}$} & \multicolumn{2}{|l|}{ Inz } \\
\hline & I & $\mathrm{nI}$ & I & $\mathrm{nI}$ & I & $\mathrm{nI}$ & I & $\mathrm{nI}$ \\
\hline N1 ampl. & -3 & -3.1 & -5.68 & -5.25 & -6.91 & -5.97 & -5.55 & -4.83 \\
\hline S.D. & 1.7 & 1.1 & 0.9 & 1.9 & 2.3 & 1.8 & 2.1 & 1.6 \\
\hline I-nI difference & -0.1 & & 0.43 & & 0.94 & & 0.72 & \\
\hline
\end{tabular}

\subsection{3. $N 2$ deflection}

3.2.3.1. Posterior N2. The second negative component of interest, namely $\mathrm{N} 2$ peaking at about $250 \mathrm{~ms}$, was larger at inion area $(\mathrm{IN} 2, \mathrm{IN} 2, \mathrm{IN} 3, \mathrm{IN} 4 ; P<0.01)$ as shown by post-hoc analyses for the significant Electrode factor $F(5,35)=8.0 ; P<0.001 ; \varepsilon=0.48$, correct. $F(2,17) ; P<0.005)$. As for P1 deflection, N2 to stimuli on a white background was larger than N2 to stimuli on a black background $(F(1,7)=8.61 ; P<0.02 ; \varepsilon=1)$, perhaps because of the greater average luminance level of patterns with black inducers on a white TV screen. As for P1, this effect did not interact with boundary completion mechanisms, since both BI and WI elicited larger N2 than BnI and WnI stimuli, respectively. At this latency level the interaction of Illusion $\times$ Hemisphere $(F(1,7)=19.72 ; P<0.003 ; \varepsilon=1)$ is significant, since $\mathrm{N} 2$ to patterns producing subjective figures was larger at left hemisphere sites $(P<0.05)$.

Fig. 5b clearly shows this left-sided activation associated with illusory contours perception: interested electrode sites are IN1, IN3, O1, OL and T5 over the left hemisphere and IN4 over the right hemisphere.

3.2.3.2. Midline N2. ANOVAs performed on $\mathrm{N} 2$ values recorded at midline electrode sites confirmed the results described above. N2 was larger at INz $(P<0.01)$ electrode site $(F(3,21)=3.49 ; P<0.034 ; \varepsilon=0.84$, correct. $F(3,18) ; P<0.05)$, and to patterns on a white background $(F(1,7)=13.61 ; P<0.01 ; \varepsilon=1)$.

\subsubsection{P3 deflection}

3.2.4.1. Posterior P300. Later ERP components showed significant effects, too. P300 recorded at posterior sites, peaking at about $390 \mathrm{~ms}$, was larger at parietal sites $(P<0.01)$ as significance of Electrode factor $F(2,14)=$ $22.02 ; \quad P<0.0000 ; \quad \varepsilon=0.80$, correct. $F(2,11) ; \quad P<$ 0.0005 ) shows. It was particularly sensitive to background colour since it was larger to black ( 8.55 $\mathrm{uV})$ background than to white $(7.81 \mathrm{uV})$ background $(F(1,7)=8.1 ; P<0.03 ; \varepsilon=1)$. This effect may be due to the fact that the previous negative deflection N2 was much larger to patterns on a white background, as mentioned above. Stimulus symmetry affected also
P300 amplitude $(F(1,7)=5.86 ; P<0.05 ; \varepsilon=1)$, which was larger to symmetrical $(8.71 \mathrm{uV})$ than to asymmetrical $(7.65 \mathrm{uV})$ patterns. Moreover, there was a significant interaction between Illusion $\times$ Symmetry $(F(1,7)=15.13 ; \quad P<0.006 ; \varepsilon=1)$. Post-hoc comparisons showed that $\mathrm{P} 300$ response to illusory configurations was larger than to non illusory configurations only when the stimuli were symmetrical $(P<0.01$; see Table 3 for means of posterior P300 amplitude.

The effect of stimulus symmetry is evident from the comparison of Fig. 7a with Fig. 7b, which show ERPs recorded at both posterior and anterior sites in response to patterns with either regular or irregular inducers, respectively.

This result suggests that inducer regularity may foster the process of boundary formation, perhaps reducing the amount of incoming unrelated visual information. The illusion/not-illusion difference was more pronounced at right hemispheric sites, as the significance of Illusion $\times$ Hemisphere $(F(1,7)=9.15 ; \quad P<0.02 ; \quad \varepsilon=1)$ and relative post-hoc comparisons $(P<0.01)$ show. The effect of hemispheric lateralisation for illusory contours perception at this late latency level can be observed in ERP waveforms displayed in Fig. $7 \mathrm{a}$.
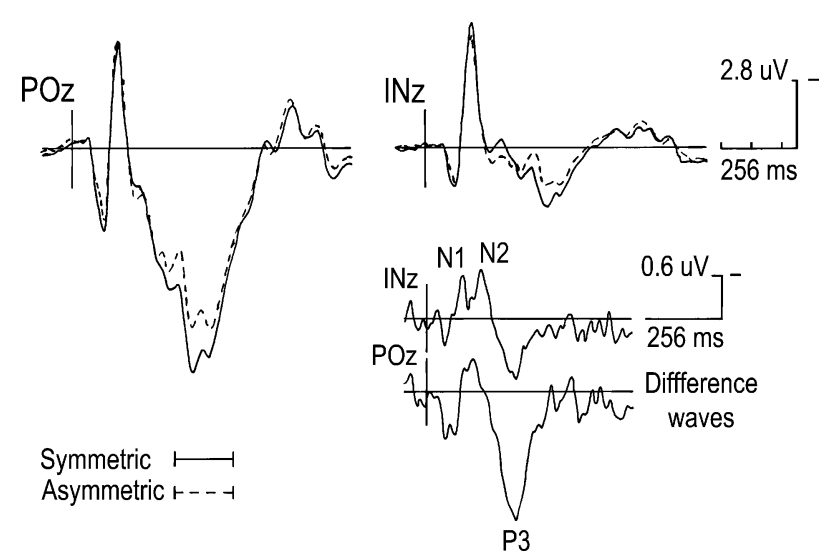

Fig. 6. Effect of stimulus symmetry. Grand-average ERPs recorded at posterior midline sites $(\mathrm{POz}$ and $\mathrm{INz})$ in response to stimuli with asymmetrical or symmetrical inducers. Symmetric stimuli elicited ERP components of greater amplitude, as can be clearly appreciated on the right in the lower part of the figure, showing the difference waves obtained subtracting ERPs to asymmetrical stimuli from ERPs to symmetrical stimuli. 
Table 3

P300 mean amplitude $(\mu \mathrm{V})$ recorded at posterior and midline sites as a function of stimulus symmetry and illusion condition

\begin{tabular}{|c|c|c|c|c|c|c|c|c|}
\hline \multirow[t]{3}{*}{ Symmetry illusion } & \multicolumn{4}{|c|}{ Posterior } & \multicolumn{4}{|c|}{ Midline } \\
\hline & \multicolumn{2}{|c|}{ Symmetrical } & \multicolumn{2}{|c|}{ Asymmetrical } & \multicolumn{2}{|c|}{ Symmetrical } & \multicolumn{2}{|c|}{ Asymmetrical } \\
\hline & I & $\mathrm{nI}$ & I & $\mathrm{nI}$ & I & $\mathrm{nI}$ & I & $\mathrm{nI}$ \\
\hline P300 ampl. & 10.2 & 7.2 & 7.5 & 7.83 & 10.88 & 8.93 & 8.46 & 8.79 \\
\hline S.D. & 4.3 & 4.4 & 4.1 & 4.5 & 2.7 & 1.8 & 3.0 & 2.2 \\
\hline I-nI difference & 3.0 & & -0.33 & & 1.95 & & -0.33 & \\
\hline
\end{tabular}

In our view, P300 data do not necessarily imply that boundary contours formation is a right-lateralised process per se, otherwise it should have been appeared in asymmetric brain activation at earlier processing stages. As mentioned above, a left-sided lateralisation was observed at earlier levels of processing, for instance at N2 level.

3.2.4.2. Midline P300. ANOVA performed on $\mathrm{P} 300$ values recorded at midline electrodes $(\mathrm{Fz}, \mathrm{Cz}, \mathrm{Pz}, \mathrm{Oz}$, Inz) showed that this positivity was larger at centralparietal sites $(P<0.01)$ as confirmed by statistical significance of Electrode factor $(F(5,35)=19.54 ; P<$ $0.001 ; \varepsilon=0.27$, correct. $F(1,9) ; P<0.005)$. Mean values are reported in Table 4.

As for posterior P300, the Symmetry $\times$ Illusion interaction was significant $(F(1,7)=27.30 ; P<0.001 ; \varepsilon=1)$. Post-hoc comparisons showed that P300 to illusory configurations was larger than to non illusory configurations $(P<0.01)$ only when inducers were symmetrical (see Table 3 for mean values).

3.2.4.3. Anterior P300. P3 response recorded at anterior sites, peaking at about $405 \mathrm{~ms}$, proved to be larger at central sites $(F(2,14)=25 ; P<0.0000 ; \varepsilon=0.80$, correct. $F(2,11) ; P<0.0005)$. This late positive response was sensitive to background luminance $(F(1,7)=10.37 ; P<$ $0.01 ; \varepsilon=1)$, being larger to stimuli on a white background than to stimuli on a black background (as shown in Fig. 8). Overall, the anterior P300 showed a significant effect of hemispheric lateralisation $(F(1,7)=$ 6.3; $P<0.04 ; \varepsilon=1)$, being larger at right than left electrode sites.

P300 was affected by stimulus Symmetry $(F(1,7)=$ 8.56; $P<0.02 ; \varepsilon=1)$, since the response to symmetrical stimuli was larger than to asymmetrical stimuli. The significant Illusion $\times$ Symmetry interaction $(F(1,7)=$ $6.11 ; P<0.04 ; \varepsilon=1)$ also showed that anterior $\mathrm{P} 3$ in illusory contours for symmetrical configurations was larger than for asymmetrical configurations, hence symmetrical inducers may facilitate the detection of illusory squares.

The significance of Illusion $\times$ Electrode interaction $(F(2,14)=5.0 ; P<0.02 ; \varepsilon=0.70$, correct. $F(1,10) ; P<$
0.05) showed that $\mathrm{P} 300$ in illusory configurations was larger than in non illusory configurations at central sites $(P<0.05)$, where it reached its maximum amplitude. The interaction of Background $\times$ Symmetry $x$ Electrode was significant, too $(F(2,14)=4.7 ; P<0.03$; $\varepsilon=0.68$, correct. $F(1,10) ; P<0.05)$. Post-hoc comparisons showed that P300 to symmetrical patterns on a white background (WS) was larger than P300 to white asymmetrical patterns $(\mathrm{WaS})$ at central $(P<0.01)$ and frontal $(P<0.05)$ sites. No significant symmetry effect was found for stimuli on a black background. Table 5 shows P300 amplitude values for these statistical comparisons.

These data suggest that stimulus symmetry had a more significant effect when there were black contours on white backgrounds or when there was greater luminance, independently of illusory contours perception.

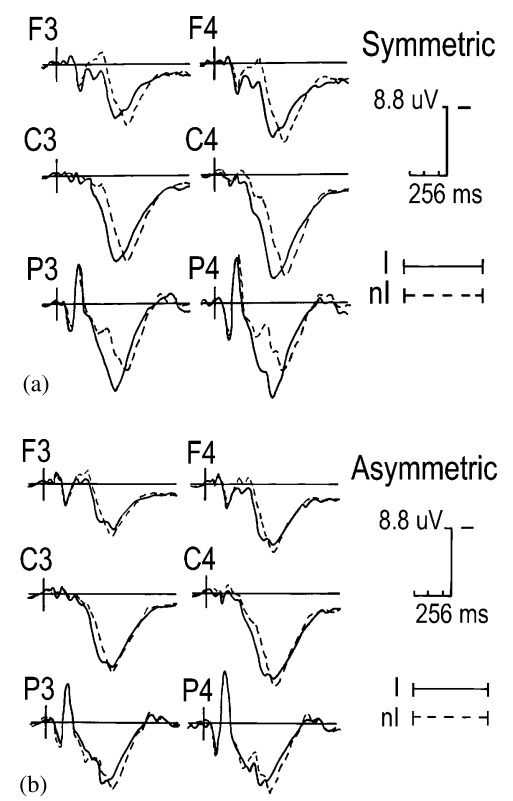

Fig. 7. Symmetry $\times$ Illusion. Grand-average ERPs recorded at homologous left and right frontal, central and parietal sites in response to symmetrical (A) or asymmetrical (B) patterns inducing (I) or not (nI) the perception of illusory contours. (A) right-sided asymmetry of the processes involved in the perception of illusory figures can be seen at parietal sites. (B) It is significant that under this condition illusory contours have little impact on P300 amplitude. 
Table 4

P300 mean amplitude $(\mu \mathrm{V})$ recorded at midline sites independent of task factors

\begin{tabular}{llcccll}
\hline Electrode & $\mathrm{Fz}$ & $\mathrm{Cz}$ & $\mathrm{Pz}$ & $\mathrm{Poz}$ & $\mathrm{Oz}$ & $\mathrm{Inz}$ \\
\hline P300 ampl. & 8.28 & 14.66 & 13.62 & 10.08 & 5.83 & 3.12 \\
S.D. & 3.8 & 5.6 & 4.1 & 4.0 & 3.9 & 2.5 \\
\hline
\end{tabular}

Yet greater luminance did not favour the boundary completion process. Behavioural data showed that error percentage was lower with dark background. This finding was consistent with P300 results. The significant Background $\times$ Illusion $\times$ Electrode interaction $(F(2,14)=4.08 ; \quad P<0.04 ; \quad \varepsilon=0.76$, correct. $F(2,11)$; $P<0.05)$ demonstrated that $\mathrm{P} 300$ response to illusory patterns was more significant when there were bright inducers on a dark background. Post-hoc comparisons for this interaction indicated that at central sites P300 to illusory configurations was larger than to non-illusory configurations on a black $(P<0.01)$ and not on a white background (see Table 6 for mean values). Fig. 9 shows brain activity related to illusory contour perception for stimuli with different type of backgrounds. The activation focus was larger, earlier and posterior for patterns with white inducers on a black background.

\section{Discussion}

The aim of the present study is to investigate the time course and the topographical distribution of brain activation, through ERP recording, during perception of patterns producing illusory contours formation vs. control patterns with a similar spatial organisation, which did not involve visual illusion. To make sure that observers paid attention to all stimuli briefly presented on the TV monitor, they were asked to press one of the two buttons at their disposal to signal the presence or

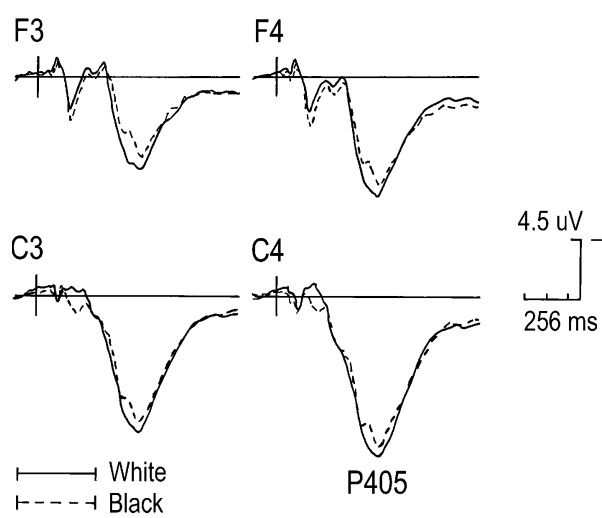

Fig. 8. Effect of Background. Grand-average ERPs recorded at homologous left and right frontal and central sites to patterns on a white or black background. Brighter backgrounds elicited larger P300 amplitude values. absence of illusory Kanizsa square. The first goal was to investigate whether there was right hemispheric lateralisation in brain activation, as suggested by several studies [14]. Secondly the study aimed at understanding if illusory contour formation occurred at early stages of visual processing pathways or if it involved associative higher level cortical regions.

ERP results showed that the occurrence of illusory contours was associated with a strong bilateral activation of lateral occipital areas at about $145 \mathrm{~ms}$ post-stimulus as indexed by the N1 deflection. This result indicates an earlier activation of visual cortex if compared to previous electrophysiological studies (i.e. 180$300 \mathrm{~ms}$ post-stimulus) [17,31,32] and it is consistent with neuroimaging data as far as neurotopographical localisation and perhaps timing of activation are concerned. Several fMRI, PET, rCBF and neurophysiological studies demonstrated an activation of extra-striate cortex (mainly V2, but also V3a, V4v, V7, V8) associated with illusory contours perception, both in humans and animals. A recent MEG study recorded an early evoked gamma activity to targets between 100 and 200 $\mathrm{ms}$, examining event-related fields and gamma activity during an active task in which observers responded to Kanizsa squares (targets) among Kanizsa triangles and non-Kanizsa figures. This finding is consistent with the timing of our earliest response to illusory contours, indexed by the amplitude modulation of $\mathrm{N} 1$ deflection.

Our data do not seem to support the hypothesis of a striate cortex (V1) involvement in illusory contour perception (as suggested by Grosof et al. [11], Hirsch et al. [14] and Leventhal et al. [22]). However, neurons signalling illusory boundaries are less numerous in V1 than in V2 [22], which might explain why a change in the activation pattern of V1 neurons is not easily detectable through scalp recordings of ERPs in humans. Our ERP data showed a significant trend, though not statistically significant, namely early sensory P1 response was affected by the presence of subjective contours (as suggested in the maps of Fig. 5a), although this effect was mostly distributed at lateral occipital sites.

Our findings for N145 component confirm that the perception of illusory boundaries takes place in the visual cortex and it is not constructed at following cognitive stages. According to classical cognitive theories illusory contours are cognitively created or "postulated' to account for perceptual gaps [9], or even unconsciously inferred to solve a perceptual problem [28], whereas Gestalt psychologists describe these phenomena as 'modal completion' operations occurring at phenomenic level [3]. Recently, it has been hypothesised that the integration of contours arises at very early stages of visual processing and that it is based on complex combinations of feedforward, feedback and lateral connections, which would produce an 'associa- 
Table 5

P300 mean amplitude $(\mu \mathrm{V})$ recorded at frontal and central sites as a function of background luminance and stimulus symmetry

\begin{tabular}{|c|c|c|c|c|c|c|c|c|}
\hline \multirow[t]{3}{*}{ Electrode background symmetry } & \multicolumn{4}{|c|}{ Frontal } & \multicolumn{4}{|c|}{ Central } \\
\hline & \multicolumn{2}{|l|}{ White } & \multicolumn{2}{|c|}{ Black } & \multicolumn{2}{|c|}{ White } & \multicolumn{2}{|l|}{ Black } \\
\hline & $\mathrm{S}$ & $\mathrm{aS}$ & $\mathrm{S}$ & $\mathrm{aS}$ & $\mathrm{S}$ & $\mathrm{aS}$ & $\mathrm{S}$ & $\mathrm{aS}$ \\
\hline P300 ampl. & 10.43 & 8.85 & 8.99 & 8.11 & 13.74 & 12.19 & 11.04 & 11.33 \\
\hline S.D. & 4.1 & 3.8 & 4.1 & 4.0 & 4.4 & 4.3 & 4.2 & 4.1 \\
\hline S-aS difference & 1.58 & & 0.88 & & 1.55 & & -0.29 & \\
\hline
\end{tabular}

tive field' integrated by long-range lateral connections [13]. Our electrophysiological data confirm this hypothesis and outline the role of edge continuity and boundary alignment in integrating visual contours.

Furthermore, our data do not provide evidence of right-lateralised neural processes underlying illusory contours perception. They show that the two cerebral hemispheres are differently activated during sensoryperceptual analysis, attentional selection and cognitive evaluation processes subserving the formation of illusory contours percept. Indeed, the illusion/not-illusion difference was more significant at left occipital area at N2 latency level (about $250 \mathrm{~ms}$ post-stimulus) and at right parietal sites at P300 level. Regarding the N2 component, which is related to attention selection mechanisms, several studies describe left hemispheric lateralisation or dominance for non-spatial feature selection at occipital scalp sites [37,38]. This negative response, recorded from lateral occipital OL and OR electrode sites in the VEP study by Sugawara and Morotomi [31], did not show hemispheric asymmetry. The task consisted in a passive viewing paradigm; attention was focused on a central fixation point and not on the (possible) subjective figure, as in the present study. In another VEP study adopting a passive viewing task, Korshunova [17] recorded a peak-to-peak N180P230 increase in amplitude at mesial occipital areas (O1 and $\mathrm{O} 2$ electrode sites) associated with illusory contour perception, along with asymmetric activation of the posterior temporal site (T5), probably near the extrastriate area (area 19).

The right-sided P300 hemispheric asymmetry, emerged in the present experiment, might be a surface reflection of higher order processes lateralisation such as perceptual grouping at global level or unified shape formation, as suggested by recent neuropsychological studies [2,4]. Evidence of hemispheric lateralisation in the processing of visual information at global/local level $[18,25,26]$ consistent with this observation has been provided both in unilaterally brain damaged patients and neurologically intact volunteers. Hence the right hemisphere is dominant in processing the global form of objects, whereas the left hemisphere is responsible for high frequency analysis and local selection of non spatial features.
Right hemisphere asymmetry was also recorded in Tallon-Baudry et al. [32], who investigated visual mechanisms of features binding analysing EEG high frequency oscillations during perception of real or illusory Kanizsa triangles and controls (no-triangles). The authors observed a non-phase locked $30-40 \mathrm{~Hz}$ component between 300 and 400 ms post-stimulus, which was stronger to the illusory triangle than to the no-triangle. At the same latency, evoked potentials showed a more positive response to the real triangle than to the illusory or the no-triangle stimulus, at right parietal sites. A relationship between P300 data and features binding processes as indexed by EEG gamma responses may be possible. According to this hypothesis, neurones responding to a shape (real or illusory) would discharge in synchrony at about $35-40 \mathrm{~Hz}$ and this oscillatory event might be a mechanism of feature binding, based on a high frequency discharge synchronisation [12,32]. The recorded activity reflects a deep and significant or widely distributed neuronal activity, deriving from visual system and perhaps from hippocampus or cingulate cortex. The same brain loci may be involved in the generation of P300, too. One of our referees advanced the intriguing hypothesis that the late right hemispheric activity found in our study might reflect the awareness of viewing an illusory object. Which is certainly very interesting and consistent with the above-mentioned EEG oscillation literature.

In our study, the finding of a larger P300 amplitude to patterns eliciting subjective figures was consistent with behavioural data indicating faster reaction times to this type of stimuli. The greater response to illusory than to non illusory configurations, might reflect a

Table 6

P300 mean amplitude $(\mu \mathrm{V})$ recorded at central sites as a function of background color and illusion condition

\begin{tabular}{lcccccc}
\hline Background illusion & \multicolumn{2}{l}{ White } & & & \multicolumn{2}{l}{ Black } \\
\cline { 2 - 3 } \cline { 5 - 6 } \cline { 5 - 6 } & $\mathrm{I}$ & $\mathrm{nI}$ & & $\mathrm{I}$ & $\mathrm{nI}$ \\
\hline P300 ampl. & 13.29 & 12.65 & & 11.99 & 10.37 \\
S.D. & 4.5 & 4.2 & & 4.2 & 4.6 \\
I-nI difference & 0.64 & & & 1.62 & \\
\hline
\end{tabular}




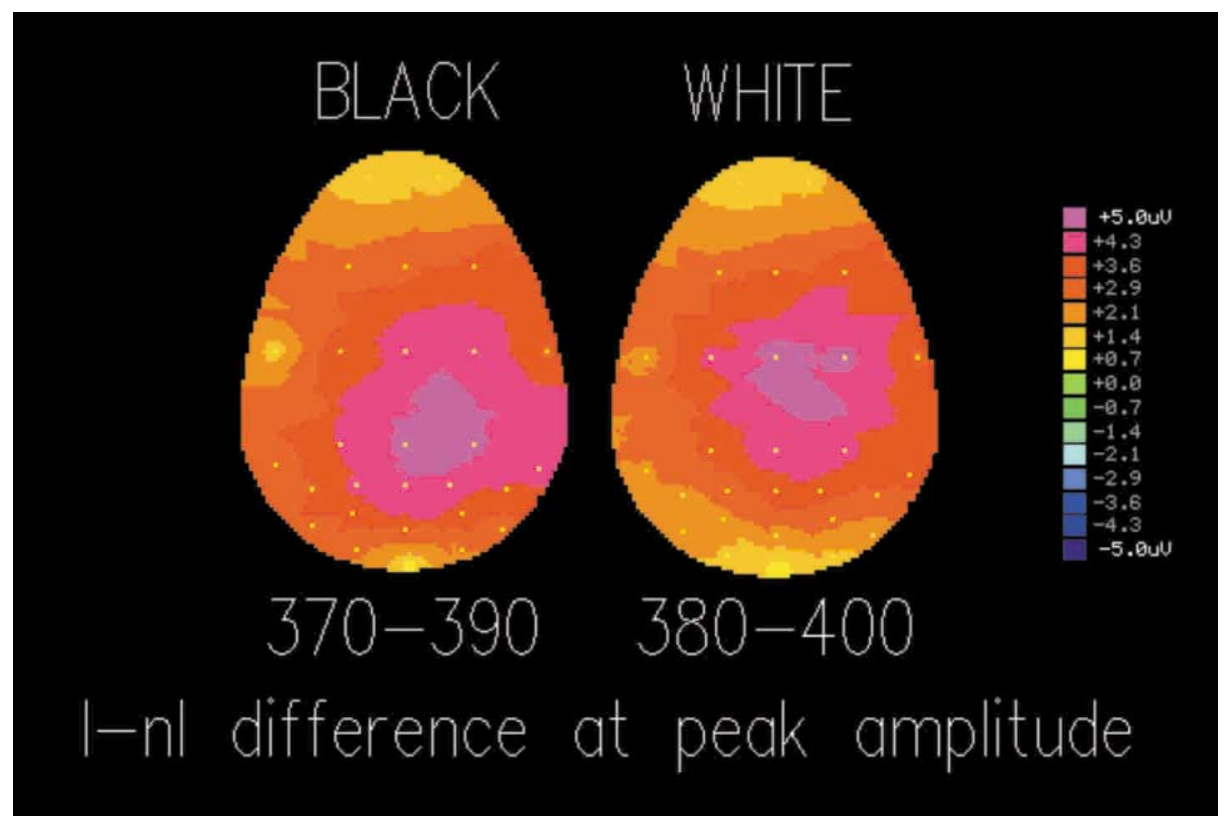

Fig. 9. Effect of illusory contours. Isocontour voltage difference-maps computed by subtracting ERPs to nI configurations from ERPs to I configurations. For black patterns the peak of the effect was reached between 370 and $390 \mathrm{~ms}$ (left), whereas for white patterns between 380 and $400 \mathrm{~ms}$.

repetition effect. Indeed, the Kanizsa square, as a consistent percept, has been repeated more often than the various non-illusory stimuli, therefore becoming more familiar. The present study is not sufficient to disentangle this factor from illusory contours perception, hence further investigation is required to draw a conclusion.

As far as the illusion effect is concerned a correlation between error percentages in behavioural performance and electrophysiological data (amplitude of P300) was recorded. In particular P300 at anterior central sites was significantly sensitive to the subjective square only when inducers were bright on dark background and on this condition depended the lowest percentages of error in task performance.

Background luminance affected ERPs amplitude significantly and interacted with boundary completion processes. Indeed, P1, N2 and P3 (at anterior sites) to black-on-white patterns were larger than to white-onblack patterns, probably because of the higher luminance level of photic stimulation. Yet the illusion effect on the amplitude of these evoked responses was similar or even larger in the latter condition; which depends on the fact that the white-on-black condition provided a better stimulation for neurones signalling boundaries, since inducing elements were bright ( $\mathrm{ON}$ response) on a dark background, whereas in the opposite condition inducers were dark (OFF response) and the less meaningful background was luminous.

A further issue is to be discussed, namely the lack of darkening effect for black illusory squares recorded by observers in our study. Psychophysical studies reported that illusory figures seem darker than the surrounding surface when inducers are white on a dark or gray background. Several authors assume that the change in luminance of subjective figures is strictly related to the formation of illusory contours of a figure [36]. A significant aspect of our study concerns the weak effect of enhanced darkening of subjective figures on a black background, due probably to our average low luminance level [30], which did not interact with boundary formation, illusion strength or clarity. As mentioned above, the illusion effect on P300 evoked response amplitude was larger in white-on-black conditions, which also led to a smaller percentage of errors. The present finding is consistent with the assumption that the illusory contours formation and occurrence of darkness (or brightness) enhancement in the centre of the display are not mutually dependent phenomena [7]. Our data indicate that the formation of illusory boundaries is affected by stimulus irregularity or asymmetry. The fact that P300 response to illusory configurations was larger than response to non illusory configurations, especially when inducers were symmetrical, indicates that inducer regularity might facilitate the process of boundaries formation. Irregular inducers may produce a certain amount of signal noise in the boundary completion process, interfering with visual neural computing.

These data suggest the primary role of inducer collinearity and edges alignment in the completion of illusory boundaries [21] at relatively early stages of visual processing. 


\section{Acknowledgements}

The authors wish to express their gratitude to Walter Gerbino for the helpful discussion on the experimental design and to Ilaria Monticone for her technical assistance and help in data collection. They also gratefully acknowledge the helpful comments of two anonymous reviewers. This research was supported by a grant from the University of Trieste to A.M. Proverbio.

\section{References}

[1] Albert MK. Parallelism and the perception of illusory contours. Perception 1993;22(5):589-95.

[2] Atchley RA, Atchley P. Hemispheric specialisation in the detection of subjective objects. Neuropsychologia 1998;36:1373-86.

[3] Bruno N, Gerbino W. Amodal completion and illusory contours. In: Petry S, Meyer G, editors. The Perception of Illusory Contours. New York: Springer, 1987:220-3.

[4] Corballis PM, Fendrich R, Shapley RM, Gazzaniga MS. Illusory contour perception and amodal boundary completion: evidence of a dissociation following callosotomy. Journal of Cognitive Neuroscience 1999;11:459-66.

[5] Coren S. Subjective contours and apparent depth. Psychological Review 1972;79:359-67.

[6] Dresp B, Grossberg S. Spatial facilitation by colour and luminance edges: boundary, surface, and attentional factors. Vision Research 1999;39:3431-43.

[7] Dresp B, Lorenceau J, Bonnet C. Apparent brightness enhancement in the Kanizsa square with and without illusory contour formation. Perception 1990;19:483-9.

[8] Ffytche DH, Zeki S. Brain activity related to the perception of illusory contours. Neuroimage 1996;3:104-8.

[9] Gregory RL. Cognitive contours. Nature 1972;238:51-2.

[10] Gregory RL, Harris JP. Illusory contours and stereo depth. Perception and Psychophysics 1974;15:411-6.

[11] Grosof DH, Shapley RM, Hawken MJ. Macaque V1 neurons can signal 'illusory' contours. Nature 1993;365:550-2.

[12] Herrmann CS, Mecklinger A, Pfeifer E. Gamma responses and ERPs in a visual classification task. Clinical Neurophysiology 1999;110:636-42.

[13] Hess R, Field D. Integration of contours: new insights. Trends in Cognitive Sciences 1999;3:480-6.

[14] Hirsch J, DeLaPaz R, Relkin RN, Victor J, Kim K, Li T, Borden $\mathrm{P}$, Rubin N, Shapley R. Illusory contours activate specific regions in human visual cortex: evidence from functional magnetic resonance imaging. Proceedings of National Academy of Sciences USA 1995;92:6469-73.

[15] Kanizsa G. Subjective contour. Scientific American 1976;234:4852.

[16] Kanizsa G. Organisation in Vision. New York: Praeger, 1979.

[17] Korshunova SG. Visual evoked potentials induced by illusory outlines (Kanizsa square). Neuroscience and Behavioural Physiology 1999;29:695-701.

[18] Lamb MR, Robertson LC, Knight RT. Component mechanisms underlying the processing of hierarchically organised patterns: inferences from patients with unilateral cortical lesions. Journal of
Experimental Psychology: Learning, Memory and Cognition 1989; $16: 471-83$.

[19] Larsson J, Amuntus K, Gulys B, Malikovic A, Zilles K, Roland PE. Neuronal correlates of real and illusory contour perception: functional anatomy with PET. European Journal of Neuroscience 1999;11:4024-36.

[20] Lesher GW. Illusory contours: toward a neurally based perceptual theory. Psychonomic Bullettin \& Review 1995;2:279-321.

[21] Lesher GW, Mingolla E. The role of edges and line-ends in illusory contour formation. Neuropsychologia 1993;33:2253-70.

[22] Leventhal AG, Wang Y, Schmolesky MT, Zhou Y. Neural correlates of boundary perception. Visual Neuroscience 1998;15:1107-18.

[23] Mendola JD, Dale AM, Fischl B, Liu AK, Tootell RB. The representation of illusory and real contours in human cortical visual areas revealed by functional magnetic resonance imaging. Journal of Neuroscience 1999;19:8560-72.

[24] Parks TE. Subjective figures: some unusual concomitant brightness effects. Perception 1980;9:239-41.

[25] Proverbio AM, Minniti A, Zani A. Electrophysiological evidence of a perceptual precedence of global vs. local visual information. Cognitive Brain Research 1998;6:321-34.

[26] Rafal R, Robertson L. The neurology of visual attention. In: Gazzaniga M, editor. The Cognitive Neurosciences. Cambridge, MA: The MIT Press, 1995:625-48.

[27] Ringach DL, Shapley R. Spatial and temporal properties of illusory contours and amodal boundary completion. Vision Research 1996;36:3037-50.

[28] Rock I. A problem-solving approach to illusory contours. In: Petry S, Meyer G, editors. The Perception of Illusory Contours. New York: Springer, 1987:62-70.

[29] Siegel S, Petry S. Evidence for independent processing of subjective contour brightness and sharpness. Perception 1991;20:233-41.

[30] Spillman L, Fuld K, Neumeyer C. Brightness matching, brightness cancellation, and increment threshold in the Ehrestein illusion. Perception 1984;13:513-20.

[31] Sugawara M, Morotomi T. Visual evoked potentials elicited by subjective contour figures. Scandinavian Journal of Psychology 1991;32:352-7.

[32] Tallon-Baudry C, Bertrand C, Delpuech C, Pernier J. Stimulus specificity of phase-locked and non-phase-locked $40 \mathrm{~Hz}$ visual responses in human. The Journal of Neuroscience 1996;16:42409.

[33] Varin D. Fenomeni di contrasto e diffusione cromatica nell'organizzazione spaziale del campo recettivo. Rivista di Psicologia 1971;65:101-28.

[34] Von der Heydt R, Petherhans E, Baumgartner G. Illusory contours and cortical neurone responses. Science 1984;224:12602.

[35] Wasserstein J, Zapulla R, Rosen J, Gerstman L, Rock D. In search of closure: subjective contour illusions, gestalt completion tests, and implications. Brain and Cognition 1987;6:1-14.

[36] Watanabe T, Oyama T. Are illusory contours a cause or a consequence of apparent differences in brightness and depth in the Kanizsa square? Perception 1988;17:513-21.

[37] Zani A, Proverbio AM. ERP signs of early selective attention effects to check size. Electroencephalography and Clinical Neurophysiology 1995;95:277-92.

[38] Zani A, Proverbio AM. Attention modulation of short latency ERPs by selective attention to conjunction of spatial frequency and location. Journal of Psychophysiology 1997;11:21-32. 